Sharif University of Technology
Scientia Iranica
Transactions E: Industrial Engineering
wCIENTIA

\title{
Vendor-managed inventory system with partial backordering for evaporating chemical raw material
}

\author{
A.A. Taleizadeh* \\ School of Industrial Engineering, College of Engineering, University of Tehran, Tehran, Iran.
}

Received 23 December 2013; received in revised form 21 July 2015; accepted 26 September 2015

\author{
KEYWORDS \\ Supply chain \\ management; \\ Inventory; \\ Partial backordering; \\ Evaporating product; \\ deterioration.
}

\begin{abstract}
Consider a supply chain including a refinery producing evaporating chemical product, an exporter, and one or some engine oil producers outside the exporter's country. The exporter uses vendor-managed inventory system implemented between refinery and exporter to decrease his/her inventory cost. This paper develops two models with partial backordering for evaporating chemical product developed in a two-layer chain including single refinery and single exporter with one product before and after utilizing vendormanaged inventory policy. Demand and partial backordering rates are deterministic and constant. A numerical example is provided to illustrate the applicability of the proposed model and solution method.
\end{abstract}

(C) 2017 Sharif University of Technology. All rights reserved.

\section{Introduction and literature review}

Vendor-managed inventory is a practice to improve the inventory management in supply chains. In this system, a vendor decides on the appropriate inventory levels of each product for all his/her retailers and the appropriate inventory policies to maintain these levels [1]. The objective of the members of VMI system is to decrease both inventory and replenishment costs in each supply chain, which benefits all members of the chain. Recently, this policy has widely been studied and developed by many researchers. For instance, Yang et al. [2] studied the effects of a distribution center in a VMI policy including single manufacturer and multi buyers. Hemmelmayr et al. [3] employed vendor-managed inventory policy for the delivery of blood products to hospitals in Eastern Austria. Yu and Huang [4] studied how a producer and its buyers interact with each other to optimize their marketing strategies, platform product configuration, and inventory management policies

*. Tel.: +98(21) 8208-4486; Fax: +98 (21) 8801-3102, E-mail address: Taleizadeh@ut.ac.ir in vendor-managed inventory system utilized in the proposed supply chain. Guan and Zhao [5] developed vendor-managed inventory system under continuous review $(r, Q)$ policy. Yao et al. [6] developed VMI system with stochastic demand and backordered shortage. Kristianto et al. [7] developed an adaptive fuzzy control application to support a vendormanaged inventory policy in a supply chain. Darwish and Odah [8] developed and utilized vendor-managed inventory policy in a supply chain including single vendor and several retailers. Egri and Vancza [9] studied the problem of coordination in supply networks including single supplier and multiple retailers where members had asymmetric information about demand and costs. Yu et al. [10] analyzed retailer selection in a vendor-managed inventory system for a manufacturer. Zanoni et al. [11] studied two issues having usual interactions in practice, which were the 'VMI with consignment' and the 'Learning Curve' policies. Chen et al. [12] studied the impact of transshipment and demand variability on distribution policies of the vendor when vendor-managed inventory strategy was used. Yu et al. [13] developed VMI policy for replenishing deteriorating raw ma- 
terial in which shortage was not permitted. Shu et al. [14] designed a logistic network using vendormanaged inventory for a company that was in charge of managing inventory for its retailers and downstream warehouses. Taleizadeh et al. [15] studied a joint optimization of price, replenishment frequency, replenishment cycle, and production rate in vendormanaged inventory system for deteriorating items. Moreover, recently, Cardnas-Barron et al. [16] reviewed the developments in EOQ models in honor of Ford Whitman Harris. Yang et al. [17] analyzed the robustness of different supply chain strategies such as VMI, electronic point of sales, e-shopping, and emergency transshipments under various uncertain environments. Kastsian and Monnigmann [18] studied robustness and stability of a supply chain in which vendor-managed inventory was used. Lee and Ren [19] examined the benefits of VMI in a global environment under periodic-review stochastic inventory policy, in which the supplier and the retailer faced uncertain exchange rate and incurred different fixed ordering costs. Almehdawe and Mantin [20] modeled a Stackelberg-game vendor-managed inventory framework with two different scenarios. In the first one, the manufacturer was the leader and in the second one, the retailer was leader. Wong et al. [21] studied how a sales rebate contract helped to achieve supply chain coordination, allowing chain members with decentralized decisions to perform a centralized decision for the whole system. Zavanella and Zanoni [22] developed an integrated inventory production model in which vendor-managed policy was used. $\mathrm{Xu}$ and Leung [23] studied a retail channel and proposed an analytical model for the members of that channel to determine the inventory policy such that the net profit of channel was maximized. Southard and Swenseth [24] studied VMI policy in a unique chain such that a firm could justify spending the money necessary to create the infrastructure to support it. Gumus et al. [25] analyzed the impacts of VMI policy and consignment inventory in a twolayer supply chain. Hariga and Al-Ahmari [26] developed integrated inventory-shelf allocation model for a stock-dependent demand of single product. The integrated model was developed for a supply chain operating under VMI policy and consignment stock agreement. Yu et al. [27] developed a Stackelberg game-theoretic model to optimize inventory, pricing, and advertising decisions in Vendor-Managed Inventory (VMI) system. Michaelraj and Shahabudeen [28] developed two supply chain models operating under VMI policy with two different objectives. In the first one, distributors had the objective of minimizing the balance payment considering the financial safety and in the second one, they had the objective of maximizing the sales with an aim to go beyond business survival. Passandideh et al. [29] developed a twolayer supply chain in which VMI and EOQ policies were used, but shortage was not permitted. Then, Passandideh et al. developed their model to a multiproduct multi-constraint case and solved it with genetic algorithm. In continuation, Cardenas Barron et al. [30] developed a more efficient algorithm to solve their proposed model. Liao et al. [31] developed a multi-objective integrated inventory-location distribution network problem operating under vendormanaged inventory policy. Chen et al. [32] studied the problem of coordinating a vertically separated distribution system under vendor-managed inventory and consignment arrangements.

There is much additional research related to VMI policy which is not introduced here. But, the interesting point is that none of the studies has developed and utilized VMI policy under partial backordering for an evaporating or deteriorating item. Thus, the aim of this research is to extend and utilize the VMI policy with partial backordering for evaporating or deteriorating items. Moreover, in this paper, a real case in petroleum industry is presented and the developed model is used to solve the problem on hand for that firm.

\section{Problem definition}

In this section, we describe a case study in which a refinery and an exporter and some retailers of an evaporating chemical product, which are engine oil producers, exist. Decisions about when and how much product should be ordered are so important. Exporter intends to use Vendor-Managed Inventory (VMI) system and asks the refinery to use VMI policy in order to decrease his/her cost.

The second characteristic of the store's situation is that if the engine oil producers order the product but cannot buy it because the exporter cannot satisfy demand of the company at appropriated time, they may do one of two things based on how much of it they still have. If they have enough of the product to wait until the exporter satisfies their demand, their order will be backordered. But if engine oil producers do not have enough of the product to wait, they will apply to another exporter in the store and will buy it from that one. In this situation, the sale at the original exporter will be lost. If we assume that a fraction of the demands can be postponed and the remaining fraction will go elsewhere, which is the case, the shortage at the original exporter will be partially backlogged, which is one of the assumptions of the proposed model.

The third characteristic of the store's situation is that the commodity exchange is evaporating raw material of engine oil factory and needs special attentions because of evaporation and its effects on 
mathematical modeling. Moreover, this paper can be used for deteriorating product instead of evaporating one, because of their similarities in assumptions and modeling.

In addition to the characteristics of the problem, it is assumed that all parameters of the problem, including evaporation, partial backordering and demand rates, and cost parameters, are constant and deterministic. In the next section, we will model the problem on hand.

\section{Modeling}

To model the problem, the following notations are used.

$q_{N} \quad$ The order quantity of exporter

$q \quad$ The order quantity of exporter in vendor-managed inventory system

$\theta \quad$ The constant rate of product evaporation

$A_{R} \quad$ The setup cost per order of the refinery

$A_{E} \quad$ The ordering cost per order of the exporter

C The deterioration cost per unit

$d \quad$ The constant demand rate of the exporter

$\mu \quad$ The partial backordering rate of the exporter

$h \quad$ The inventory holding cost of exporter in a period per unit per unit time

$b_{N} \quad$ The maximum level of backordering shortage of exporter

$b \quad$ The maximum level of backordering shortage of exporter in vendor-managed inventory system

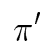

The fixed cost of lost sale of exporter per unit

$\hat{\pi} \quad$ The cost of shortage of exporter per unit per time

$T_{N} \quad$ The time cycle

$T \quad$ The time cycle in vendor-managed inventory system

$F_{N} \quad$ The percentage of cycle length with positive inventory

$F \quad$ The percentage of cycle length with positive inventory in vendor-managed inventory system

$T C E_{N} \quad$ Inventory cost of the exporter in vendor-managed inventory system

TCE Inventory cost of the exporter in vendor-managed inventory system

$T C R_{N} \quad$ Inventory cost of the refinery
$T C R \quad$ Inventory cost of the refinery in vendor-managed inventory policy

$T C_{N} \quad$ The total cost before vendor-managed inventory policy

TC The total cost of vendor-managed inventory policy

Because of demand and deterioration, during $[0, F T]$, the inventory level drops to zero. Thus, the differential equation shown in Eq. (1) shows the change in the inventory level during $[0, F T]$.

$$
\frac{d I_{1}(t)}{d t}=-\theta I_{1}(t)-d, \quad 0 \leq t \leq F T .
$$

Therefore, we have:

$$
I_{1}(t)=\frac{d}{\theta}\left(e^{\theta(F T-t)}-1\right) .
$$

Furthermore, at time $F T$, shortage occurs and the inventory level starts dropping below zero such that, finally, the backordered and lost sale quantities are respectively:

$$
\begin{aligned}
& b=\mu d(1-F) T, \\
& L=(1-\mu)(1-F) d T .
\end{aligned}
$$

Therefore, we have:

$$
I_{\max }=I_{1}(0)=\frac{d}{\theta}\left(e^{\theta F T}-1\right) .
$$

Since $Q=I_{\max }+b$, we have:

$$
q=\frac{d}{\theta}\left(e^{\theta F T}-1\right)+\mu d(1-F) T .
$$

The total cost of system for the cycle time, $T$, is made up of the exporter's ordering cost, refinery's ordering cost, the holding cost of product stored in exporter's warehouse in a period, deterioration cost, and cost of shortage. The exporter's holding cost is:

$$
\begin{aligned}
h & \times \int_{0}^{F T} I_{1}(t)=h \times \int_{0}^{F T} \frac{d}{\theta}\left(e^{\theta(F T-t)}-1\right) d t \\
& =-\left.\frac{d}{\theta^{2}}\left(e^{\theta(F T-t)}+\theta t\right)\right|_{0} ^{F T}=\frac{h d}{\theta^{2}}\left[e^{\theta F T}-\theta F T-1\right] .
\end{aligned}
$$

Moreover, the fixed cost of exporter is $A_{E}$ and the deterioration cost will be:

$$
C\left(I_{1}(0)-d F T\right)=C\left[\frac{d}{\theta}\left(e^{\theta(F T)}-1\right)-d F T\right],
$$

and the back-ordered and lost sale costs are respectively:

$$
\begin{aligned}
& \frac{\pi \mu d(1-F)^{2} T^{2}}{2}, \\
& \pi^{\prime} d(1-\mu)(1-F) T .
\end{aligned}
$$




\subsection{Analysis of inventory costs}

Before utilizing vendor-managed inventory system, the total cost function of the exporter and the total cost function of refinery are respectively shown in Eqs. (11) and (12). Then, the total cost of the chain is shown in Eq. (13):

$$
\begin{aligned}
T C E_{N}= & \frac{1}{T}\left(A_{E}+\frac{d}{\theta^{2}}\left[e^{\theta F_{N} T_{N}}-\theta F_{N} T_{N}-1\right]\right. \\
& +C\left[\frac{d}{\theta}\left(e^{\theta F_{N} T_{N}}-1\right)-d F_{N} T_{N}\right] \\
& +\frac{\hat{\pi} \mu d\left(1-F_{N}\right)^{2} T_{N}^{2}}{2} \\
& \left.+\pi^{\prime} d(1-\mu)\left(1-F_{N}\right) T_{N}\right), \\
T C R_{N}= & \frac{A_{R}}{T}, \\
T C_{N}= & T C E_{N}+T C R_{N}=\frac{1}{T}\left(A_{R}+A_{E}\right. \\
+ & \left.\pi^{\prime} d(1-\mu)\left(1-F_{N}\right) T_{N}\right) . \\
+ & \frac{d}{\theta^{2}}\left[e^{\theta F_{N} T_{N}}-\theta F_{N} T_{N}-1\right] \\
+ & \left.C \frac{d}{\theta}\left(e^{\theta F_{N} T_{N}}-1\right)-d F_{N} T_{N}\right] \\
+ & \frac{\pi^{2} d\left(1-F_{N}\right)^{2} T_{N}^{2}}{2} \\
& \\
& \\
& \\
& \\
& \\
&
\end{aligned}
$$

Using approximation of the Taylor series expansion, $e^{\theta F_{N} T_{N}}=1+\theta F_{N} T_{N}+\frac{\left(\theta F_{N} T_{N}\right)^{2}}{2}$, we have:

$$
\begin{aligned}
T C E_{N}= & \frac{1}{T_{N}}\left(A_{E}+\frac{(\theta C+h) d\left(F_{N} T_{N}\right)^{2}}{2}\right. \\
& +\frac{\hat{\pi} \mu d\left(1-F_{N}\right)^{2} T_{N}^{2}}{2} \\
& \left.+\pi^{\prime} d(1-\mu)\left(1-F_{N}\right) T_{N}\right), \\
T C R_{N}= & \frac{A_{R}}{T_{N}}, \\
T C_{N}= & \frac{1}{T_{N}}\left(A_{R}+A_{E}+\frac{(\theta C+h) d\left(F_{N} T_{N}\right)^{2}}{2}\right.
\end{aligned}
$$

\subsection{Solution method}

The exporter's inventory cost in Eq. (14) is a function of $T$ and $F$. Thus, global optimal values of $T$ and $F$ can be obtained by taking the partial derivative of Eq. (14) with respect to $T$ and $F$; then setting them equal to zero (in Appendix'A, we prove that $T^{*}$ and $F^{*}$ give a global optimal solution).

$$
\frac{\partial T C E_{N}}{\partial T_{N}}=\frac{-A_{E}}{T_{N}^{2}}+\frac{(h+C \theta) d F_{N}^{2}}{2}+\frac{\hat{\pi} \mu d\left(1-F_{N}\right)^{2}}{2} .
$$

Then, we have:

$$
T_{N}^{*}\left(F_{N}\right)=\sqrt{\frac{2 A_{E}}{(h+C \theta) d F_{N}^{2}+\hat{\pi} \mu d\left(1-F_{N}\right)^{2}}} .
$$

and:

$$
\begin{aligned}
\frac{\partial T C E_{N}}{\partial F_{N}}= & (h+C \theta) d F_{N} T_{N}-\hat{\pi} \mu d T_{N}\left(1-F_{N}\right) \\
& -\pi^{\prime} d(1-\mu) .
\end{aligned}
$$

Finally:

$$
F_{N}=\frac{\mu \hat{\pi} T_{N}+\pi^{\prime}(1-\mu)}{(h+C \theta+\mu \hat{\pi}) T_{N}} .
$$

Substituting $F_{N}^{*}$ into $T_{N}^{*}\left(F_{N}^{*}\right)$ yields (see Appendix B):

$$
T_{N}=\sqrt{\frac{2 A_{E}(h+C \theta+\mu \hat{\pi})-d\left(\pi^{\prime}(1-\mu)\right)^{2}}{\mu \hat{\pi}(h+C \theta) d}} .
$$

Shortage quantity before utilizing vendormanaged inventory policy can be calculated as follows (see Eq. (3)):

$$
b_{N}^{*}=\mu d\left(1-F_{N}^{*}\right) T_{N}^{*} .
$$

Moreover, the ordering quantity before utilizing vendor-managed inventory policy using approximation of the Taylor series expansion in Eq. (6) will change to:

$$
q_{N}^{*}=d\left(F_{N} T_{N}+\frac{\theta\left(F_{N} T_{N}\right)^{2}}{2}\right)+\mu d\left(1-F_{N}\right) T_{N} .
$$

The optimal values of decision variables $T$ and $F$ of the exporter's total cost shown in Eq. (14) can be obtained using Eqs. (20) and (21) only if $\mu$ satisfies the inequality given in Eq. (24) (see Appendix C).

$$
\mu>\mu_{N}^{*}=1-\frac{\sqrt{2 A_{E}(h+C \theta) d}}{d \pi^{\prime}} .
$$


Then, this solution is optimal if this condition is met and $\mu^{*}>0$. If the condition is met but $\mu^{*}<0$, the cost of the optimal partial backordering solution must be compared with the cost of not stocking. To summarize: If $\mu>\mu^{*}>0$ (where $\mu^{*}$ is given in Eq. (24)), then the optimal partial backordering solution is given by Eqs. (20) and (21); if, however, $\mu^{*}<0$, the cost of that solution must be compared with the cost of not stocking at all. If $\mu<\mu^{*}$, the optimal solution is either to use the classic EOQ model or not to stock the item at all, whichever costs less [33$36]$.

But, when vendor-managed inventory policy is used, since refinery should pay the exporter costs, Eqs. (14) to (16) will change to:

$$
\begin{aligned}
T C E= & 0 \\
T C R= & \frac{1}{T}\left(A_{R}+A_{E}+\frac{h d}{\theta^{2}}\left[e^{\theta F T}-\theta F T-1\right]\right. \\
& +C\left[\frac{d}{\theta}\left(e^{\theta F T}-1\right)-d F T\right] \\
& \left.+\frac{\hat{\pi} \mu d(1-F)^{2} T^{2}}{2}+\pi^{\prime} d(1-\mu)(1-F) T\right),(26) \\
T C= & C E+T C R=\frac{1}{T}\left(A_{R}+A_{E}\right. \\
+ & \frac{h d}{\theta^{2}}\left[e^{\theta F T}-\theta F T-1\right] \\
+ & C\left[\frac{d}{\theta}\left(e^{\theta F T}-1\right)-d F T\right] \\
+ & \left.\frac{\hat{\pi} \mu d(1-F)^{2} T^{2}}{2}+\pi^{\prime} d(1-\mu)(1-F) T\right)
\end{aligned}
$$

Using approximation of the Taylor series expansion, we have:

$$
\begin{aligned}
T C R= & \frac{1}{T}\left(A_{R}+A_{E}+\frac{(\theta C+h) d(F T)^{2}}{2}\right. \\
& \left.+\frac{\hat{\pi} \mu d(1-F)^{2} T^{2}}{2}+\pi^{\prime} d(1-\mu)(1-F) T\right) \\
T C= & \frac{1}{T}\left(A_{R}+A_{E}+\frac{(\theta C+h) d(F T)^{2}}{2}\right. \\
& \left.+\frac{\hat{\pi} \mu d(1-F)^{2} T^{2}}{2}+\pi^{\prime} d(1-\mu)(1-F) T\right)
\end{aligned}
$$

Once again, since the total inventory cost in Eq. (28) is a function of $T$ and $F$, global optimal values of $T$ and $F$ can be obtained by taking the partial derivative of Eq. (28) with respect to $T$ and $F$, then setting them equal to zero (as we mentioned in the previous section, in Appendix A, we prove that $T^{*}$ and $F^{*}$ give a global optimal solution):

$$
\frac{\partial T C}{\partial T}=-\frac{\left(A_{R}+A_{E}\right)}{T^{2}}+\frac{d(C \theta+h) F^{2}}{2}+\frac{\hat{\pi} \mu d(1-F)^{2}}{2}
$$

Then, we have:

$$
T^{*}(F)=\sqrt{\frac{2\left(A_{R}+A_{E}\right)}{d(h+C) F^{2}+\hat{\pi} \mu d(1-F)^{2}}},
$$

and:

$$
\frac{\partial T C}{\partial F}=(h+C \theta) d F T-\hat{\pi} \mu d T(1-F)-\pi^{\prime} d(1-\mu) .
$$

Finally:

$$
F^{*}=\frac{\pi^{\prime}(1-\mu)+\mu \hat{\pi} T}{(h+C \theta+\mu \hat{\pi}) T} .
$$

Substituting $F^{*}$ into $T^{*}\left(F^{*}\right)$, same as when vendormanaged inventory system is not utilized, yields:

$$
T^{*}=\sqrt{\frac{2\left(A_{R}+A_{E}\right)(h+C \theta+\mu \hat{\pi})-d\left(\pi^{\prime}(1-\mu)\right)^{2}}{(\mu \hat{\pi})(h+C \theta) d}},
$$

the shortage and order quantities after utilizing vendormanaged inventory policy are respectively:

$$
\begin{aligned}
& b^{*}=\mu d\left(1-F^{*}\right) T^{*}, \\
& q^{*}=d\left(F T+\frac{\theta(F T)^{2}}{2}\right)+\mu d(1-F) T .
\end{aligned}
$$

Same as the previous case, Eqs. (33) and (34) give the optimal values of $T$ and $F$ for the cost function in Eq. (14) only if $\mu$ satisfies the inequality given in Eq. (29).

$$
\mu>\mu^{*}=1-\frac{\sqrt{2\left(A_{R}+A_{E}\right)(h+C \theta) d}}{d \pi^{\prime}} .
$$

Utilizing the solution method proposed by Pentico et al. [37], the following solution procedure can be developed to determine the optimal values of $T$ and $F$ when VMI policy is used:

1. Calculate $\mu^{*}=1-\frac{\sqrt{2\left(A_{R}+A_{E}\right)(h+C \theta) d}}{d \pi^{\prime}}$; 
2. If $\mu \geq \mu^{*}$, go to Step 3. If $\mu<\mu^{*}$, determine the optimal cost of 'no stockouts' and compare this with the cost of losing all demands, $\pi^{\prime} d$, to determine whether it is optimal to allow no stockouts or all stockouts. If $\sqrt{2\left(A_{R}+A_{E}\right) d\left(h+C^{\prime} \theta\right)} \leq \pi^{\prime} d$, then $F^{*}=1, T^{*}=\sqrt{\frac{2\left(A_{R}+A_{E}\right)}{d(h+C \theta)}}, q^{*}=d T^{*}$, and $b^{*}=0$. If $\sqrt{2\left(A_{R}+A_{E}\right) d\left(h+C^{\prime} \theta\right)}>\pi^{\prime} d$, then $T^{*}=\infty$, $F^{*}=q^{*}=0$ and Stop;

3. Using Eq. (33) and (34), calculate the couple of $\left(T^{*}, F^{*}\right)$ and go to step 4 . If $\mu^{*}<0$, compute $T C\left(F^{*}, T^{*}\right)$ using Eq. (29); if $\psi\left(F^{*}, T^{*}\right)<\pi^{\prime} d$, go to step 4. Otherwise, $T^{*}=\infty$, and $F^{*}=q^{*}=0$. Stop;

4. Determine the optimal value of shortage and order quantities using Eqs. (35) and (36).

If we would like to solve an EOQ model with partial backordering for deteriorating item (ignoring VMI policy), we should use $A_{S}=0$ in the described solution method and all equations will be derived for when VMI policy is ignored.

\section{Numerical example}

In order to illustrate the above solution procedure, let us consider an inventory system with the following data:

$$
\begin{aligned}
& A_{R}=100, \quad A_{E}=100, \quad h=3, \quad d=2,000, \\
& C=100, \quad \theta=0.005, \quad \pi^{\prime}=1, \quad \hat{\pi}=2 .
\end{aligned}
$$

4.1. First example: $\mu=0.5$

Using Eq. (37), we have $\mu=0.5$ :

\section{- Step 1.}

$$
\begin{aligned}
\mu^{*} & =1-\frac{\sqrt{2\left(A_{R}+A_{E}\right)(h+C \theta) d}}{d \pi^{\prime}} \\
& =1-\frac{\sqrt{2(100+100)(3+100 \times 0.005) 2000}}{2000(1)} \\
& =0.1633
\end{aligned}
$$

- Step 2. Since $\mu=0.5>\mu^{*}=0.1633$, go to Step 3;

- Step 3. Using Eqs. (34) and (33), we have:

$$
\begin{aligned}
T^{*} & =\sqrt{\frac{2(100+100)(3+100 \times 0.005+0.5 \times 2)-2000(1(1-0.5))^{2}}{(0.5 \times 2)(3+100 \times 0.005) 2000}} \\
& =0.4309 \\
F^{*} & =\frac{1(1-0.5)+2(0.5) 0.4309}{(3+100 \times 0.005+2 \times 0.005) 0.4309}=0.48 .
\end{aligned}
$$

- Step 4. Using Eqs. (35) and (36), we have:

$$
\begin{aligned}
b^{*}= & 0.5 \times 2000 \times(1-0.48) \times 0.4309=224.0689 \\
q^{*}= & 2000\left(0.43 \times 0.4309+\frac{0.005(0.43 \times 0.4309)^{2}}{2}\right) \\
& +0.5 \times 2000(1-0.48) 0.4309=638.0366
\end{aligned}
$$

\subsection{Second example $\mu=0.1$}

- Step 1. From the first step of the first example, we know $\mu^{*}=0.1633$;

- Step 2. Since $\mu=0.1<\mu^{*}=0.1633$, the optimal cost of 'no stockouts' and the cost of losing all demands should be compared. Since the optimal cost of 'no stockouts' $\sqrt{2\left(A_{R}+A_{E}\right) d\left(h+C^{\prime} \theta\right)}=$ $\sqrt{2(100+100) 2000(3+100 \times 0.005)}=1673.32$ is less than the cost of losing all demands $\pi^{\prime} d=$ $1 \times 2000=2000$, we have:

$$
\begin{aligned}
F^{*} & =1, \\
T^{*} & =\sqrt{\frac{2\left(A_{R}+A_{E}\right)}{d(h+C \theta)}}=\sqrt{\frac{2(100+100)}{2000(3+100 \times 0.005)}} \\
& =0.2390
\end{aligned}
$$

and:

$$
q^{*}=d T^{*}=2000 \times 0.2390=478.0914 .
$$

\section{Conclusion}

In this paper, a supply chain including a refinery producing evaporating chemical product and a distributer who exports the products of refinery, with and without vendor-managed inventory policy, is studied. According to the observations of real case, the partial backordering is assumed where all parameters of the model are constant and deterministic. The closedform solutions for decision variables, including optimal period length, order and shortage quantities of evaporating item under vendor-managed inventory policy, after proofing the concavity of objective function, are derived. Two numerical examples are provided to illustrate the applicability of the proposed model and solution method. The result shows that vendor-managed inventory decreases cost in all conditions and is more beneficial for the coordination system. For future research, considering stochastic partial backordering or demand rates and several competing refineries or exporters, considering pricing and revenue management topic, using time-dependent partial backordering rate or using contacts between the partners can enhance the developed model.

\section{References}

1. Yu, Y., Chu, F. and Chen, H. "A Stackelberg game and its improvement in a VMI system with a man- 
ufacturing vendor", European Journal of Operational Research, 192, pp. 929-948 (2009).

2. Yang, L., Ng, C.T. and Cheng, T.C.E. "Evaluating the effects of distribution centers on the performance of vendor-managed inventory systems", European Journal of Operational Research, 201, pp. 112-122 (2010).

3. Hemmelmayr, V., Doerner, K.F., Hartl, R.F. and Savelbergh, M.W.P. "Vendor managed inventory for environments with stochastic product usage", European Journal of Operational Research, 202, pp. 686695 (2010).

4. Yu, Y. and Huang, G.Q. "Nash game model for optimizing market strategies, configuration of platform products in a Vendor Managed Inventory (VMI) supply chain for a product family", European Journal of Operational Research, 206, pp. 361-373 (2010).

5. Guan, R. and Zhao, X. "On contracts for VMI program with continuous review (r,Q) policy", European Journal of Operational Research, 207, pp. 656-667 (2010).

6. Yao, Y., Dong, Y. and Dresner, M. "Managing supply chain backorders under vendor managed inventory: An incentive approach and empirical analysis", European Journal of Operational Research, 203, pp. 350-359 (2010).

7. Kristianto, Y., Helo, P., Jiao, J. and Sandhu, M. "Adaptive fuzzy vendor managed inventory control for mitigating the Bullwhip effect in supply chains", European Journal of Operational Research, 216, pp. 346-355 (2012).

8. Darwish, M.A. and Odah, O.M. "Vendor managed inventory model for single-vendor multi-retailer supply chains", European Journal of Operational Research, 204, pp. 473-484 (2010).

9. Egri, P. and Vancza, J. "A distributed coordination mechanism for supply networks with asymmetric information", European Journal of Operational Research, 226, pp. 452-460 (2013).

10. Yu, Y., Hong, Z., Zhang, L.L., Liang, L. and Chu, C. "Optimal selection of retailers for a manufacturing vendor in a vendor managed inventory system", European Journal of Operational Research, 225, pp. 273-284 (2013).

11. Zanoni, S., Jaber, M.Y. and Zavanella, L.E. "Vendor Managed Inventory (VMI) with consignment considering learning and forgetting effects", International Journal of Production Economics, 140, pp. 721-730 (2012).

12. Chen, X., Hao, G., Li, X. and Yin, K.F.C. "The impact of demand variability and transshipment on vendor's distribution policies under vendor managed inventory strategy", International Journal of Production Economics, 139, pp. 42-48 (2012).

13. Yu, Y., Wang, Z. and Liang, L. "A vendor managed inventory supply chain with deteriorating raw materials and products", International Journal of Production Economics, 136, pp. 266-274 (2012).
14. Shu, J., Li, Z., Shen, H., Wu, T. and Zhong, W. "A logistics network design model with vendor managed inventory", International Journal of Production Economics, 135, pp. 754-761 (2012).

15. Taleizadeh, A.A., Noori-daryan, M. and CárdenasBarrón, L.E. "Joint optimization of price, replenishment frequency, replenishment cycle and production rate in vendor managed inventory system with deteriorating items", International Journal of Production Economics, 159, pp. 285-295 (2015).

16. Cárdenas-Barrón, L.E., Chung, K.J., Treviño-Garza, G. "Celebrating a century of the economic order quantity model in honor of Ford Whitman Harris", International Journal of Production Economics, 155, pp. 1-7 (2014).

17. Yang, T., Wen, Y.F. and Wang, F.F. "Evaluation of robustness of supply chain information-sharing strategies using a hybrid Taguchi and multiple criteria decisionmaking method", International Journal of Production Economics, 134, pp. 458-466 (2011).

18. Kastsian, D. and Monnigmann, M. "Optimization of a vendor managed inventory supply chain with guaranteed stability and robustness", International Journal of Production Economics, 131, pp. 727-735 (2011).

19. Lee, J.Y. and Ren, L. "Vendor-managed inventory in a global environment with exchange rate uncertainty", International Journal of Production Economics, 130, pp. 169-174 (2011).

20. Almehdawe, E. and Mantin, B. "Vendor managed inventory with a capacitated manufacturer and multiple retailers: Retailer versus manufacturer leadership", International Journal of Production Economics, 128, pp. 292-302 (2010).

21. Wong, W.K., Qi, J. and Leung, S.Y.S. "Coordinating supply chains with sales rebate contracts and vendormanaged inventory", International Journal of Production Economics, 120, pp. 151-161 (2009).

22. Zavanella, L. and Zanoni, S. "A one-vendor multibuyer integrated production-inventory model: The 'Consignment Stock' case", International Journal of Production Economics, 118, pp. 225-232 (2009).

23. Xu, K. and Leung, M.T. "Stocking policy in a two-party vendor managed channel with space restrictions", International Journal of Production Economics, 117, pp. 271-285 (2009).

24. Southard, P.B. and Swenseth, S.R. "Evaluating vendor-managed inventory (VMI) in non-traditional environments using simulation", International Journal of Production Economics, 116, pp. 275-287 (2008).

25. Gumus, M., Jewkes, E.M. and Bookbinder, J.H. "Impact of consignment inventory and vendor-managed inventory for a two-party supply chain", International Journal of Production Economics, 113, pp. 502-517 (2008).

26. Hariga, M.A. and Al-Ahmari, A. "An integrated retail space allocation and lot sizing models under vendor 
managed inventory and consignment stock arrangements", Computers \& Industrial Engineering, 64, pp. 45-55 (2013).

27. Yu, Y., Huang, G.Q. and Liang, L. "Stackelberg gametheoretic model for optimizing advertising, pricing and inventory policies in vendor managed inventory (VMI) production supply chains", Computers \& Industrial Engineering, 57, pp. 368-382 (2009).

28. Michaelraj, L.A. and Shahabudeen, P. "Replenishment policies for sustainable business development in a continuous credit based vendor managed inventory distribution system", Computers \& Industrial Engineering, 56, pp. 260-266 (2009).

29. Pasandideh, S.H.R., Akhavan Niaki, S.T. and Roozbeh $\mathrm{Nia}, \mathrm{A}$. "An investigation of vendor-managed inventory application in supply chain: the EOQ model with shortage", International Journal of Advanced Manufacturing Technology, 49, pp. 329-339 (2010).

30. Cardenas-Barron, L.E., Trevino-Garza, G. and Wee, H.M. "A simple and better algorithm to solve the vendor managed inventory control system of multiproduct multi-constraint economic order quantity model", Expert Systems with Applications, 39, pp. 3888-3895 (2012).

31. Liao, S.H., Hsieh, C.L. and Lai, P.J. "An evolutionary approach for multi-objective optimization of the integrated location-inventory distribution network problem in vendor-managed inventory", Expert Systems with Applications, 38, pp. 6768-6776 (2011).

32. Chen, J.M., Lin, I.C. and Cheng, H.L. "Channel coordination under consignment and vendor managed inventory in a distribution system", Transportation Research Part E, 46, pp. 831-843 (2010).

33. Taleizadeh, A.A. and Pentico, D.W., "An economic order quantity model with partial backordering and known price increase", European Journal of Operational Research, 28, pp. 516-525 (2013a).

34. Taleizadeh, A.A., Pentico, D.W., Jabalameli, M.S. and Aryanezhad, M.B. "An EOQ problem under partial delayed payment and partial backordering", Omega, 41(2), pp. 354-368 (2013b).

35. Taleizadeh, A.A., Pentico, D.W., Jabalameli, M.S. and Aryanezhad, M.B. "An economic order quantity model with multiple partial prepayments and partial backordering", Mathematical and Computer Modelling, 57(3-4), pp. 311-323 (2013c).

36. Taleizadeh, A.A., Pentico, D.W., Aryanezhad, M.B. and Ghoreyshi, M. "An economic order quantity model with partial backordering and a special sale price", European Journal of Operational Research, 221(3), pp. 571-583 (2012).

37. Pentico, D.W., Drake, M.J. and Toews, C. "The deterministic EOQ with partial backordering: A new approach", European Journal of Operational Research, 194, pp. 102-113 (2009).

\section{Appendix A}

\section{Proof of the optimality of the solutions}

(Eqs. (20), (21), (33), and (34))

Using the method proposed by Pentico et al. [37], we can prove that Eqs. (20), (21), (33), and (34) are global optimal, although the cost functions, which are shown in Eqs. (16) and (29), are not convex. The cost function can be rewritten as follows:

$$
\begin{aligned}
T C(F, T)= & \frac{\lambda_{0}}{T}+\left(\lambda_{1} F^{2}-2 \lambda_{2} F+\lambda_{2}\right) T \\
& -\lambda_{3} F+\lambda_{3},
\end{aligned}
$$

where:

$$
\begin{aligned}
& \lambda_{0}=A>0, \\
& \left(\mathrm{No} \mathrm{VMI}: A=A_{E} ; \mathrm{VMI}: A=A_{R}+A_{E}\right), \\
& \lambda_{1}=\frac{(\theta C+h+\mu \hat{\pi}) d}{2}>0, \\
& \lambda_{2}=\frac{\hat{\pi} \mu d}{2}>0, \\
& \lambda_{3}=\pi^{\prime} d(1-\mu)>0 .
\end{aligned}
$$

Note that all the $\lambda_{i} \mathrm{~s}$ are positive. We can rewrite Eq. (A.1) as follows:

$$
T C(F, T)=\frac{\lambda_{0}}{T}+\operatorname{Tr}(F)+q(F),
$$

where:

$$
r(F)=\lambda_{1} F^{2}-2 \lambda_{2} F+\lambda_{2} .
$$

and:

$$
q(F)=-\lambda_{3} F+\lambda_{3} .
$$

Our objective is to establish the condition under which Eq. (A.6) has a unique interior minimizer. Differentiating Eq. (A.6) with respect to $T$ yields:

$$
\frac{\partial T C}{\partial T}=-\frac{\lambda_{0}}{T^{2}}+r(F)
$$

which equals zero if and only if $T$ satisfies:

$$
T=T^{*}(F)=\sqrt{\frac{\lambda_{0}}{r(F)}} .
$$

Note that this is the same result, with appropriate change of notation, given in Eqs. (21) and (33). Since the discriminant of $r(F)$ is negative, $r(F)$ has no roots. Thus, $r(F)$ is either all-positive or all-negative. Since $r(0)=\lambda_{4}>0, r(F)$ is strictly positive in $[0,1]$. Thus, Eq. (A.10) gives, for each $F$, a unique $T^{*}=T^{*}(F)$ 
that minimizes the cost function given by Eq. (A.7). Substituting the expression for $T^{*}(F)$ in Eq. (A.10) into $T C(F, T)$ given by Eq. (A.6) gives:

$$
T C(F) \equiv T C\left(T^{*}(F), F\right)=2 \sqrt{\lambda_{0} r(F)}+q(F)
$$

which represents minimal possible cost for each value of $F$. Note that $T C(F)$ is continuous; thus, on the compact interval $[0,1]$, it has one or more local minima, the smallest of which will be the global minimum of the cost function [37]. To find these minima, take the first and second derivatives of $T C(F)$ with respect to $F$, yielding:

$$
\begin{aligned}
& T C^{\prime}(F)=\sqrt{\lambda_{0}} \frac{r^{\prime}(F)}{r(F)^{\frac{1}{2}}}+q^{\prime}(F), \\
& T C^{\prime \prime}(F)=\frac{\sqrt{\lambda_{0}}\left[2 r^{\prime \prime}(F) r(F)-\left(r^{\prime}(F)\right)^{2}\right]}{2 r(F)^{\frac{3}{2}}} .
\end{aligned}
$$

Note that $T C^{\prime}(F)$, which is, with the change in notation, same as $\frac{\partial T C(F, T)}{\partial F}$ given in Eqs. (19) and (32), is continuous and satisfies $T C^{\prime}(0)<0$.

$$
\begin{aligned}
& r^{\prime}(F)=2 \lambda_{1} F-2 \lambda_{2}, \\
& q^{\prime}(F)=-\lambda_{3}, \\
& r^{\prime \prime}(F)=2 \lambda_{1}, \\
& q^{\prime \prime}(F)=0, \\
& T C^{\prime}(0)=-\left(\sqrt{\lambda_{0}} \frac{\lambda_{3}}{\sqrt{\lambda_{2}}}+\lambda_{3}\right)<0 .
\end{aligned}
$$

The second derivative, $T C^{\prime \prime}(F)$, given in Eq. (A.13) is as below:

$$
\begin{aligned}
T C^{\prime \prime} & (F) \\
& =\frac{\sqrt{\lambda_{0}}\left[4 \lambda_{1}\left(\lambda_{1} F^{2}-2 \lambda_{2} F+\lambda_{2}\right)-\left(2 \lambda_{1} F-2 \lambda_{2}\right)^{2}\right]}{2 r(F)^{\frac{3}{2}}} \\
& =\frac{\sqrt{\lambda_{0}}\left[-8 \lambda_{1} \lambda_{2} F+4 \lambda_{1} \lambda_{2}-4 \lambda_{2}^{2}+8 \lambda_{1} \lambda_{2} F\right]}{2 r(F)^{\frac{3}{2}}} \\
& =\frac{\sqrt{\lambda_{0}}\left[4 \lambda_{1} \lambda_{2}-4 \lambda_{2}^{2}\right]}{2 r(F)^{\frac{3}{2}}}>0,
\end{aligned}
$$

which is positive for all $F \mathrm{~s}$, because $\lambda_{1}=\frac{(\theta C+h+\mu \hat{\pi}) d}{2}$ and $\lambda_{2}=\frac{\hat{\pi} \mu d}{2}$, meaning $4 \lambda_{1} \lambda_{2}>4 \lambda_{2}^{2}$.

\section{Appendix B}

Deriving the optimal value of period length shown in Eqs. (31) and (33)

To proof Eq. (21), from Eq. (18), we know:

$$
T_{N}^{*^{2}}=\frac{2 A_{E}}{(h+C \theta) d F_{N}^{2}+\hat{\pi} \mu d\left(1-F_{N}\right)^{2}} .
$$

Moreover, from Eq. (20) we have:

$$
F_{N}=\frac{\mu \hat{\pi} T_{N}+\pi^{\prime}(1-\mu)}{(h+C \theta+\mu \hat{\pi}) T_{N}} .
$$

Replacing $F_{N}$ and $1-F_{N}$ in Eq. (B.1) by:

$$
F_{N}=\frac{\mu \hat{\pi} T_{N}+\pi^{\prime}(1-\mu)}{(h+C \theta+\mu \hat{\pi}) T_{N}}
$$

and:

$$
1-F_{N}=\frac{(h+C \theta) T_{N}-\pi^{\prime}(1-\mu)}{(h+C \theta+\mu \hat{\pi}) T_{N}},
$$

respectively yields in Eqs. (B.3) and (B.4) as shown in Box B.1. Finally, we have:

$$
T_{N}=\sqrt{\frac{2 A_{E}(h+C \theta+\mu \hat{\pi})-d\left(\pi^{\prime}(1-\mu)\right)^{2}}{(\mu \hat{\pi})(h+C \theta) d}} .
$$

\section{Appendix $\mathrm{C}$}

\section{Deriving lower limit of partial backordering rate}

Since $T^{*}$ for the partial backordering model must be at least as large as $T^{*}$ for the basic EOQ model, firstly, we should determine $T^{*}$ for the basic EOQ model. Thus, if we consider $F=1$, then Eq. (14) (the total cost when there is no shortage) will change to:

$$
T C E_{N}=\frac{A_{E}}{T_{N}}+\frac{(\theta C+h) d T_{N}}{2},
$$

and we will have:

$$
\begin{aligned}
& \frac{d T C E_{N}}{d T_{N}}=-\frac{A_{E}}{T_{N}^{2}}+\frac{h d}{2}+\frac{C \theta d}{2}, \\
& \frac{d^{2} T C E_{N}}{d^{2} T_{N}}=\frac{2 A_{E}}{T_{N}^{3}} \geq 0 .
\end{aligned}
$$

Since the second derivative is positive for all values of $T>0$, the objective function shown in Eq. (C.1) is convex and setting the first derivative equal to zero will yield the optimal period length shown in Eq. (C.4).

$$
T_{N}^{*}=\sqrt{\frac{2 A_{E}}{d(h+C \theta)}} .
$$

Now, considering $T_{N}^{*}$ for the partial backordering model greater than $T_{N}^{*}$ for the basic EOQ model, we have:

$$
\frac{2 A_{E}(h+C \theta+\mu \hat{\pi})-d\left(\pi^{\prime}(1-\mu)\right)^{2}}{(\mu \hat{\pi})(h+C \theta) d} \geq \frac{2 A_{E}}{d(h+C \theta)},
$$




$$
T_{N}^{*^{2}}=\frac{2 A_{E}}{(h+C \theta) d\left(\frac{\mu \hat{\pi} T_{N}+\pi^{\prime}(1-\mu)}{(h+C \theta+\mu \hat{\pi}) T_{N}}\right)^{2}+\hat{\pi} \mu d\left(\frac{(h+C \theta) T_{N}-\pi^{\prime}(1-\mu)}{(h+C \theta+\mu \hat{\pi}) T_{N}}\right)^{2}} .
$$

Then:

$$
\begin{aligned}
T_{N}^{*^{2}}= & \frac{2 A_{E}(h+C \theta+\mu \hat{\pi})^{2} T_{N}^{2}}{(h+C \theta) d\left(\mu \hat{\pi} T_{N}+\pi^{\prime}(1-\mu)\right)^{2}+\hat{\pi} \mu d\left((h+C \theta) T_{N}-\pi^{\prime}(1-\mu)\right)^{2}} \\
= & \frac{2 A_{E}(h+C \theta+\mu \hat{\pi})^{2} T_{N}^{*^{2}}}{(h+C \theta) d\left(\mu \hat{\pi} T_{N}+\pi^{\prime}(1-\mu)\right)^{2}+\hat{\pi} \mu d\left((h+C \theta) T_{N}-\pi^{\prime}(1-\mu)\right)^{2}} \\
= & \frac{2 A_{E}(h+C \theta+\mu \hat{\pi})^{2} T_{N}^{*^{2}}}{(h+C \theta) d\left(\left(\mu \hat{\pi} T_{N}\right)^{2}+\left(\pi^{\prime}(1-\mu)\right)^{2}+2 \beta \hat{\pi} \pi^{\prime}(1-\mu) T_{N}\right)} \\
= & \frac{\left.+\hat{\pi} \mu d\left((h+C \theta) T_{N}\right)^{2}+\left(\pi^{\prime}(1-\mu)\right)^{2}-2(h+C \theta) \pi^{\prime}(1-\mu) T_{N}\right)}{(h+C \theta) d\left(\left(\mu \hat{\pi} T_{N}\right)^{2}+\left(\pi^{\prime}(1-\mu)\right)^{2}\right)+2 \mu \hat{\pi} \pi^{\prime}(h+C \theta) d(1-\mu) T_{N}} \\
= & \frac{\left.+\hat{\pi} \mu d\left((h+C \theta) T_{N}\right)^{2}+\left(\pi^{\prime}(1-\mu)\right)^{2}\right)-2 \hat{\pi} \pi^{\prime} \mu(h+C \theta) d(1-\mu) T_{N}}{(\mu \hat{\pi})(h+C \theta) d(\mu \hat{\pi}+h+C \theta) T_{N}^{2}+(h+C \theta+\mu \hat{\pi}) d\left(\pi^{\prime}(1-\mu)\right)^{2}} \\
= & \frac{2 A_{E}(h+C \theta+\mu \hat{\pi}) T_{N}^{*^{2}}}{(\mu \hat{\pi})(h+C \theta) d T_{N}^{2}+d\left(\pi^{\prime}(1-\mu)\right)^{2}}=\frac{2 A_{E}(h+C \theta+\mu \hat{\pi})-d\left(\pi^{\prime}(1-\mu)\right)^{2}}{(\mu \hat{\pi})(h+C \theta) d} .
\end{aligned} .
$$

\section{Box B.1}

$\frac{2 A_{E}(h+C \theta+\mu \hat{\pi})}{(\mu \hat{\pi})(h+C \theta) d}-\frac{2 A_{E}}{d(h+C \theta)} \geq$

$\frac{\left(\pi^{\prime}(1-\mu)\right)^{2}}{(\mu \hat{\pi})(h+C \theta)}$

$\frac{2 A_{E}(h+C \theta+\mu \hat{\pi})}{\mu \hat{\pi}(h+C \theta) d}-\frac{2 \mu \hat{\pi} A_{E}}{\mu \hat{\pi} d(h+C \theta)} \geq \frac{\left(\pi^{\prime}(1-\mu)\right)^{2}}{(\mu \hat{\pi})(h+C \theta)}$,

$\frac{2 A_{E}(h+C \theta)}{\mu \hat{\pi}(h+C \theta) d} \geq \frac{d\left(\pi^{\prime}(1-\mu)\right)^{2}}{\mu \hat{\pi}(h+C \theta) d}$,

$\frac{2 A_{E}}{\mu \hat{\pi} d} \geq \frac{\left(\pi^{\prime}(1-\mu)\right)^{2}}{\mu \hat{\pi}(h+C \theta)}$

$\frac{2 A_{E}}{d} \geq \frac{\left(\pi^{\prime}(1-\mu)\right)^{2}}{(h+C \theta)}$,

$$
\mu \geq 1-\frac{\sqrt{2 A_{E}(h+C \theta) d}}{d \pi^{\prime}} .
$$

\section{Biography}

Ata Allah Taleizadeh is an Assistant Professor in the School of Industrial Engineering at University of Tehran, in Iran. He received his $\mathrm{PhD}$ degree in Industrial Engineering from Iran University of Science and Technology. Moreover, he received his BSc and MSc degrees both in Industrial Engineering from Azad University of Qazvin and Iran University of Science and Technology, respectively. His research interest areas include inventory control and production planning, pricing and revenue optimization, and uncertain programming. He has published several papers and book chapters in reputable journals and he serves as the editor/editorial board member in a number of international journals. 